\title{
METZLER PHILOSOPHIE LEXIKON
}

Begriffe und Definitionen 


\title{
METZLER PHILOSOPHIE LEXIKON
}

Begriffe und Definitionen

\author{
Herausgegeben von \\ Peter Prechtl und Franz-Peter Burkard
}

Verlag J.B. Metzler Stuttgart · Weimar 


\title{
Inhaltsverzeichnis
}

\author{
Vorwort Seite $V$ \\ Autoren- und Kürzelverzeichnis Seite VII \\ Abkürzungen Seite IX \\ Logische und mathematische Symbole Seite XI
}

Die Deutsche Bibliothek - CIP-Einheitsaufnahme

Metzler-Philosophie-Lexikon : Begriffe und Definitionen

/ hrsg. von Peter Prechtl und Franz-Peter Burkard.

- Stuttgart ; Weimar: Metzler, 1996

ISBN 978-3-476-01257-9

NE: Prechtl, Peter [Hrsg.]; Philosophie-Lexikon

ISBN 978-3-476-01257-9

ISBN 978-3-476-03561-5 (eBook)

DOI 10.1007/978-3-476-03561-5

Dieses Werk einschließlich aller seiner Teile ist urheberrechtlich geschützt. Jede Verwertung außerhalb der engen Grenzen des Urheberrechtsgesetzes ist ohne

Zustimmung des Verlages unzulässig und strafbar. Das gilt insbesondere für Vervielfältigungen, Übersetzungen, Mikroverfilmungen und die Einspeicherung und Verarbeitung in elektronischen Systemen.

(C) 1996 Springer-Verlag GmbH Deutschland

Ursprünglich erschienen bei J. B. Metzlersche Verlagsbuchhandlung und Carl Ernst Poeschel Verlag GmbH in Stuttgart 1996 


\section{Vorwort}

Das Lexikon Philosophie ist als Arbeitsmittel für die erste Orientierung innerhalb der philosophischen Begrifflichkeit konzipiert. Es soll den Studierenden der Philosophie (auch Studienanfängern), Wissenschaftlern anderer Fachgebiete und den philosophisch Interessierten ermöglichen, sich innerhalb der verschiedenen Gebiete der Philosophie die nötige begriffliche Übersicht zu verschaffen. Um diesem Anspruch zu genügen, muß das Lexikon so umfassend wie möglich sein, ohne seine Handlichkeit als einbändiges Nachschlagewerk zu verlieren. Angesichts der Tatsache, $\mathrm{da} ß$ man es in der Philosophie weder mit monolithischen Lehrmeinungen noch mit fixiertem oder fixierbarem Wissen zu tun hat, lassen sich beide Ziele nicht einfach auf einen Nenner bringen. Für jede Begriffserläuterung galt es, eine hinreichend klare Darstellung ohne unzulässige Verkürzung zu finden. Für die Auswahl der Begriffe galt es, die Überrepräsentierung bestimmter Spezialgebiete zu vermeiden und das gesamte Spektrum philosophischer Fragestellungen abzudecken. Es liegt auf der Hand, daß ein solches Unternehmen nicht allein nach streng systematischen Gesichtspunkten zu bewerkstelligen ist.

Das Lexikon umfaßt den Bereich der abendländischen Philosophie von der Antike bis zur Gegenwart. Hinzukommen Grundbegriffe der chinesischen und indischen Philosophie. Einschränkungen bezüglich sogenannter Spezialphilosophien waren aus den oben genannten Gründen notwendig.

So wie sich um jeden "größeren « philosophischen Begriff eine ganze Problemstellung konzentriert, der dieser Begriff seine Bedeutung verdankt, so ist auch mit jeder Problemstellung ein Spektrum an philosophischen Reflexionen verbunden - entsprechend vielfältig sind die Antworten. Bei einzelnen Autoren haben zahlreiche Termini eine spezielle Ausformung erhalten. In solchen Fällen haben wir uns auf die für eine Position grundlegenden Begriffe konzentriert, die den Einstieg in das Werk eines Philosophen ermöglichen und von denen aus sich die weiteren Begriffe erschlieBen lassen. Das Lexikon kann nicht die Beschäftigung mit dem Autor ersetzen, sondern bestenfalls erste Hilfestellungen geben. Da philosophisches Denken immer auch einen Prozeß darstellt, haben wir versucht, die Bezüge herzustellen, in denen sich der betreffende Begriff bewegt, und ebenso, die Bedeutungsverschiebungen sichtbar zu machen. Auch an dieser Stelle waren Beschränkungen aus den genannten Gründen nicht zu vermeiden. Ein Großteil der Begriffe fügt sich nicht der definitorischen Festlegung, sondern wird erst in der historischen Ausfaltung hinreichend erkennbar; begriffsgeschichtliche Gesichtspunkte lassen sich immer auch als eigene Unterscheidungsgeschichte rekonstruieren. Im Lexikon waren hier Akzente historischer und systematischer Art zu setzen. Die Einbußen hinsichtlich der historischen Vielfalt der Begriffsdi- mensionen versucht das Lexikon durch den Verweis auf kontroverse Verständnisse und zumindest die wichtigsten Bedeutungsdifferenzen zu kompensieren. Für umfassendere Darstellungen philosophischer Begriffe und Fragestellungen verweisen wir auf die folgenden Werke, die natürlich auch in den Artikeln des Lexikons zur Anwendung kamen: auf das Historische Wörterbuch der Philosophie, hrsg. von J. Ritter und K. Gründer, auf die Enzyklopädie Philosophie und Wissenschaftstheorie, hrsg. von J. Mittelstraß, auf die Europäische Enzyklopädie zu Philosophie und Wissenschaften, hrsg. von H. J. Sandkühler. Für die Darstellung von einzelnen Philosophen verweisen wir auf das Metzler Philosophenlexikon.

Begriffe aus Einzelwissenschaften wie Physik, Mathematik, Sprachwissenschaft, Psychologie, Soziologie, Biologie sind im Lexikon vertreten, soweit sie Eingang in philosophische Kontexte gefunden haben und Bestandteil philosophischer Argumentation geworden sind.

Die Artikel geben die Werke der für den behandelten Begriff repräsentativen Autoren an und bieten, soweit es angebracht erscheint, einführende oder weiterführende Literatur neueren Datums, die den Einstieg in die dem Begriff zugehörige Thematik ermöglichen soll.

Die einzelnen Begriffserläuterungen sollen jeweils für sich selbst verständlich sein, ohne daß der Leser durch zahlreiche Verweise zu ständigem Weiterblättern genötigt wäre; daraus resultieren Redundanzen solcherart, daß Begriffe in jeweils anderem Zusammenhang nochmals erläutert werden. Die vorhandenen Verweise ( $)$ sind ein Angebot für den Leser, dem thematischen Bezug innerhalb des Lexikons nachzugehen. Wo es aus Gründen der Verständlichkeit geboten war, wurde mitunter der Erläuterung eines Begriffes mehr Platz eingeräumt, als es dessen systematischer Stellenwert hätte erwarten lassen.

*3

An dieser Stelle gilt unser ausdrücklicher Dank dem Verlag, speziell Bernd Lutz, der diese Konzeption mitgetragen hat. Er war es auch, der das Projekt und damit auch die Herausgeber mit entsprechendem Druck vorangetrieben hat. Bisweilen bedurfte es eines äußeren Zwanges, über unseren "philosophischen Schatten « zu springen und uns für eine Fassung eines Artikels zu entscheiden, den wir nur zu gerne im $\mathrm{x}$-fachen Umfang gebracht hätten.

Danken möchten wir auch den Mitarbeitern des Metzler-Verlags, Ute Hechtfischer, Norbert Retlich und Oliver Schütze für ihre engagierte Arbeit in verschiedenen Phasen des Projektes.

Carmen-Marion Herrmann und Ruth Dommaschk möchten wir herzlich für mühevolle redaktionelle $\mathrm{Ar}$ beit danken. 
Michael Quante, Münster, möchten wir namentlich erwähnen. Er hat nicht nur zahlreiche eigene Artikel ver$\mathrm{faßt}$, sondern in spontaner und engagierter Weise weitere Mitautoren gewonnen. Für die Herausgeber war es eine angenehme Erfahrung, wie Herr Quante das Anliegen des Lexikons uneigennützig zu seiner eigenen Sache machte und zum Gelingen des Projekts beigetragen hat.

Einen ganz persönlichen Dank habe ich an Gerhard Gehrke, Max Karges und Manfred Voith zu richten, die mir mit sämtlichen Geräten und unter denkwürdigen Umständen geholfen haben, als mein Computer ausgerechnet in der Endphase der Produktion seine technische bzw. elektronische Intelligenz aufgab und seine Mitarbeit verweigerte (PP).

Berlin/Würzburg, im Oktober 1995
Peter Prechtl Franz-Peter Burkard 


\section{Autoren- und Kürzelverzeichnis}

\begin{tabular}{|c|c|c|c|}
\hline AA & Andreas Arndt, Berlin & GOS & Gottfried Schwitzgebel, Mainz \\
\hline $\mathrm{AB}$ & Andreas Bartels, Gießen & GS & Georg Scherer, Oberhausen \\
\hline $\mathrm{AC}$ & Andreas Cremonini, Basel & GSO & Gianfranco Soldati, Tübingen \\
\hline $\mathrm{AE}$ & Achim Engstler, Münster & HB & Harald Berger, Graz \\
\hline AG & Alexander Grau, Berlin & $\mathrm{HDH}$ & Han-Ding Hong, Düsseldorf \\
\hline AK & André Kieserling, Bielefeld & $\mathrm{HG}$ & Helmut Glück, Bamberg \\
\hline $\mathrm{AN}$ & Armin Nassehi, Münster & HGR & Horst Gronke, Berlin \\
\hline AR & Alexander Riebel, Würzburg & HL & Hilge Landweer, Berlin \\
\hline BBR & Birger Brinkmeier, Münster & HND & Herta Nagl-Docekal, Wien \\
\hline $\mathrm{BC}$ & Bernadette Collenberg, Münster & HPS & Helke Pankin-Schappert, Mainz \\
\hline BD & Bernhard Debatin, Berlin & IR & Ines Riemer, Hamburg \\
\hline BG & Bernward Gesang, Kusterdingen & JA & Johann S. Ach, Münster \\
\hline BI & Bernhard Irrgang, Dresden & $\mathrm{JC}$ & Jürgen Court, Köln \\
\hline BK & Bernd Kleimann, Tübingen & JH & Jörg Hardy, Münster \\
\hline $\mathrm{BKO}$ & Boris Kositzke, Tübingen & JK & Jörg Klawitter, Würzburg \\
\hline BL & Burkhard Liebsch, Bochum & JM & Jörg F. Maas, Hannover \\
\hline BR & Boris Rähme, Berlin & JP & Jörg Pannier, Münster \\
\hline BS & Berthold Suchan, Gießen & JQ & Josef Quitterer, Innsbruck \\
\hline $\mathrm{BZ}$ & Bernhard Zimmermann, Allensbach & JR & Josef Rauscher, Mainz \\
\hline $\mathrm{CA}$ & Claudia Albert, Berlin & JRO & Johannes Rohbeck, Berlin \\
\hline CI & Christian Iber, Berlin & JS & Joachim Söder, Würzburg \\
\hline $\mathrm{CJ}$ & Christoph Jäger, Leipzig & JSC & Jörg Schmidt, München \\
\hline CK & Christian Kanzian, Innsbruck & $\mathrm{KDZ}$ & Klaus-Dieter Zacher, Berlin \\
\hline CL & Cornelia Liesenfeld, Augsburg & $\mathrm{KE}$ & Klaus Eck, Würzburg \\
\hline CLK & Clemens Kauffmann, Lappersdorf & KH & Kai-Uwe Hellmann, Berlin \\
\hline $\mathrm{CM}$ & Claudius Müller, Nehren & KHG & Karl-Heinz Gerschmann, Münster \\
\hline $\mathrm{CO}$ & Clemens Ottmers, Tübingen & KHL & Karl-Heinz Lembeck, Trier \\
\hline $\mathrm{CP}$ & Cristina de la Puente, Stuttgart & KJG & Klaus-Jürgen Grün, Frankfurt a.M. \\
\hline $\mathrm{CS}$ & Christian Schröer, München & KRL & Karl-Reinhard Lohmann, Witten \\
\hline CSE & Clemens Sedmak, Innsbruck & $\mathrm{KS}$ & Kathrin Schulz, Würzburg \\
\hline $\mathrm{CZ}$ & Christian Zeuch, Münster & $\mathrm{KSH}$ & Klaus Sachs-Hombach, Magdeburg \\
\hline DG & Dorothea Günther, Würzburg & LG & Lutz Geldsetzer, Düsseldorf \\
\hline DGR & Dorith Grugel, Münster & LR & Leonhard Richter, Würzburg \\
\hline $\mathrm{DH}$ & Detlef Horster, Hannover & MA & Mauro Antonelli, Graz \\
\hline DHB & Daniela Hoff-Bergmann, Bremen & MB & Martin Beisler, Gerbrunn \\
\hline DIK & Dietmar Köveker, Frankfurt a.M. & MBI & Marcus Birke, Münster \\
\hline DK & Dominic Kaegi, Heidelberg & MBO & Marco Bonato, Tübingen \\
\hline DKÖ & Dietmar Köhler, Witten & MD & Max Deeg, Würzburg \\
\hline DL & Dorothea Lüddeckens, Veitshöchheim & $\mathrm{ME}$ & Michael Esfeld, Münster \\
\hline DP & Dominik Perler, Göttingen & MFM & Martin F. Meyer, Münster \\
\hline $\mathrm{EE}$ & Eva Elm, Berlin & MK & Matthias Kunz, München \\
\hline EJ & Eva Jelden, Berlin & MKL & Martin Kleinsorge, Aachen \\
\hline $\mathrm{EF}$ & Elisabeth Fink, Berlin & MKO & Mathias Koßler, Mainz \\
\hline EM & Ekkehard Martens, Hamburg & ML & Mark Lekarew, Berlin \\
\hline ER & Eberhard Rüddenklau, Staufenberg & MM & Matthias Maring, Karlsruhe \\
\hline EWG & Eckard Wolz-Gottwald, Davensberg & $\mathrm{MN}$ & Marcel Niquet, Neu-Isenburg \\
\hline EWL & Elisabeth Weisser-Lohmann, Bochum & MQ & Michael Quante, Münster \\
\hline FG & Frank Grunert, Münster & MR & Mathias Richter, Rottenburg \\
\hline FPB & Franz-Peter Burkard, Würzburg & MRM & Marie-Luise Raters-Mohr, Münster \\
\hline GK & Georg Kneer, Münster & MS & Manfred Stöckler, Bremen \\
\hline GKB & Gudrun Kühne-Bertram, Ochtrup & MSI & Mark Siebel, Hamburg \\
\hline GL & Georg Lohmann, Berlin & MSU & Martin Suhr, Hamburg \\
\hline GM & Georg Mildenberger, Tübingen & MW & Markus Willaschek, Münster \\
\hline GME & Günther Mensching, Hamburg & MWÖ & Matthias Wörther, München \\
\hline GMO & Georg Mohr, Münster & NM & Norbert Meuter, Berlin \\
\hline GN & Guido Naschert, Tübingen & OFS & Orrin F. Summerell, Bochum \\
\hline
\end{tabular}


PE Peter Eisenhardt, Frankfurt a.M.

PCL Peter Ch. Lang, Frankfurt a.M.

PK Peter Kunzmann, Würzburg

PN Peter Nitschke, Münster

PP Peter Prechtl, München/Berlin

RD Ruth Dommaschk, Würzburg

RDÜ Renate Dürr, Karlsruhe

RE Rolf Elberfeld, Würzburg

REW Ruth Ewertowski, Stuttgart

RH Reiner Hedrich, Gießen

RHI Reinhard Hiltscher, Stegaurach

RK Reinhard Kottmann, Münster

RL Rudolf Lüthe, Schaan

RM Reinhard Mehring, Berlin

RP Roland Popp, Bremen

RS Regina Srowig, Würzburg

RTH Robert Theis, Strassen

RW Raymund Weyers, Köln

SD Steffen Dietzsch, Hagen

SIK Simone Koch, Bochum

SP Stephan Pohl, Dresden

TB Thomas Bausch, Berlin

TBL Thomas Blume, Dresden

TF Thomas Friedrich, Würzburg
TG Thomas Grundmann, Bonn

TH Thomas Hammer, Frankfurt a.M.

TK Thomas Kisser, München

TM Thomas Mormann, Unterhaching

TN Thomas Noetzel, Marburg

UB Ulrich Baltzer, München

UM Ulrich Metschl, Bayreuth

VG Volker Gerhardt, Berlin

VM Verena Mayer, München

VP Veit Pittioni, Innsbruck

WAM Walter Mesch, Tübingen

WB Wilhelm Baumgartner, Würzburg

WH Wolfram Hinzen, Bern

WJ Werner Jung, Duisburg

WK Wulf Kellerwessel, Aachen

WL Winfried Löffler, Innsbruck

WM Wolfgang Meckel, Butzbach

WN Wolfgang Neuser, Heidelberg

WP Wolfgang Pleger, Cochem/Dohr

WS Werner Schüßler, Trier

WST Wolfgang Struck, Göttingen

WSU Wolfgang Schulz, Tübingen

WvH Wolfram von Heynitz, Weiburg 


\section{Abkürzungen}

$\begin{array}{ll}\text { a. } & \text { Articulus } \\ \text { Abh. } & \text { Abhandlung(en) } \\ \text { Anm. } & \text { Anmerkung } \\ \text { Aufl. } & \text { Auflage } \\ \text { Bd.,Bde. } & \text { Band, Bände } \\ \text { Beih. } & \text { Beiheft(e) } \\ \text { Diss. } & \text { Dissertation } \\ \text { eingel. } & \text { eingeleitet } \\ \text { Einl. } & \text { Einleitung } \\ \text { Erg.Bd. } & \text { Ergänzungsband } \\ \text { erl. } & \text { erläutert } \\ \text { Frg. } & \text { Fragment } \\ \text { Fs. } & \text { Festschrift } \\ \text { GW, Ges. Werke } & \text { Gesammelte Werke } \\ \text { Hdb. } & \text { Handbuch } \\ \text { Hg., hg. } & \text { Herausgeber, herausgegeben } \\ \text { Jb. } & \text { Jahrbuch } \\ \text { Jg. } & \text { Jahrgang } \\ \text { Kap. } & \text { Kapitel } \\ \text { Nachdr. } & \text { Nachdruck } \\ \text { ND } & \text { Neudruck } \\ \text { NF. } & \text { Neue Folge } \\ \text { q., qu. } & \text { Quaestio } \\ \text { S. } & \text { Sutras } \\ \text { Suppl. } & \text { Supplement } \\ \text { U. } & \text { Upanișad } \\ \text { u.ö. } & \text { und öfter } \\ \text { übers. } & \text { übersetzt } \\ \text { V. } & \text { Veda } \\ \text { Zs. } & \text { Zeitschrift(en) } \\ & \end{array}$

\section{Abkürzungen von Einzelwerken}

HWPh.

Historisches Wörterbuch der Philosophie. Hg. v. J. Ritter u. K. Gründer. Basel $1971 \mathrm{ff}$.

\section{Aristoteles}

an. pr.

an. post.

de an.

Eth. Nic.

Met.

Hegel

Enc.

Logik

Phänom.

Analytica priora

Analytica posteriora

De anima

Ethica Nicomachea

Metaphysik

Encyklopädie der philosophischen Wissenschaften $\left({ }^{3} 1830\right)$

Wissenschaft der Logik (1812/ 1816)

Phänomenologie des Geistes

$$
\text { (1807) }
$$

Rechtsphil. Grundlinien der Philosophie des Rechts (1821)

$\begin{array}{ll}\text { Kant } & \\ \text { GMS } & \text { Grundlegung zur Metaphysik der } \\ & \text { Sitten } \\ \text { KpV } & \text { Kritik der praktischen Vernunft } \\ \text { KrV } & \text { Kritik der reinen Vernunft } \\ \text { KU } & \text { Kritik der Urteilskraft } \\ \text { Proleg. } & \begin{array}{l}\text { Prolegomena zu einer jeden Meta- } \\ \text { physik... }\end{array}\end{array}$

Thomas v. Aquin

S.th.

Summa theologiae

Wittgenstein

Phil. Unters. Philosophische Untersuchungen

Tract. Tractatus logico-philosophicus

\section{Abkürzungen von Werkausgaben und Sammlungen}

Descartes

Oeuvres

Oeuvres, I-XII, hg.Adam/Tannery, Paris 1897-1910, bzw. I-XI, 1964 1974

Fichte

Ges. Ausg. Gesamtausgabe der Bayerischen Akademie der Wissenschaften. Hg. R. Lauth und H. Jacob. Stuttgart-Bad Cannstatt $1964 \mathrm{ff}$.

Hegel

Ges. Werke Gesammelte Werke, Hamburg $1968 \mathrm{ff}$.

Sämtl. Werke Sämtliche Werke, Hg. Glockner, Stuttgart 1927-1939

Husserl

Hua.

Husserliana. Den Haag $1950 \mathrm{ff}$.

Kant

Akad.-Ausg. Gesammelte Schriften, hg. Königl. Preußische Akademie der Wissenschaften, Berlin $1902 \mathrm{ff}$.

Marx/Engels

MEGA

Historisch-kritische Gesamtausgabe. Frankfurt/Berlin/Moskau 1927-1935 (unvollständig)

MEW Werke. Berlin 1956-1968

PG

J. P. Migne (Hg.): Patrologiae cursus completus. Series I: Ecclesia graeca. Paris 1857-1912

PL J. P. Migne (Hg.): Patrologiae cursus completus. Series II: Ecclesia latina. Paris 1841-1864 
Schelling

Hist.-krit.Ausg. Historisch-kritische Ausgabe.

Hg. H. M. Baumgartner u. a. Stuttgart-Bad Cannstatt $1976 \mathrm{ff}$.

Sämtl. Werke
Stoiker

SVF

Vorsokratiker

VS
Stoicorum veterum fragmenta. $\mathrm{Hg}$. J. v. Arnim. Leipzig 1903-1924

Die Fragmente der Vorsokratiker. Hg. H. Diels/W. Kranz ${ }^{6} 1951$ (und weitere Aufl.) 


\section{Logische und mathematische Symbole}
A, B, C
Zeichen für Mengen oder für Aussagenkonstante
$\mathrm{a}, \mathrm{b}, \mathrm{c}$
Zeichen für Elemente oder für Individuenkonstante
F, G, H
Zeichen für Prädikate
$\mathrm{X}, \mathrm{Y}, \mathrm{Z}$
Zeichen für Individuenvariable
gdw.
genau dann, wenn
p
wird zur Kennzeichnung einer propositionalen Aussage verwendet, z. B.: »ich behaupte, daß die Straße naß ist $«$ - $»$ ich behaupte, daß p «, oder »es ist wahr, daß $\mathrm{p} «$
$\mathrm{p}$ und $\mathrm{q}$
in der Aussagenlogik Zeichen für die Teilsätze, die durch die Junktoren verknüpft werden.
$\varepsilon \quad$ es ist/es hat
$\varepsilon^{\prime} \quad$ es ist nicht/hat nicht
affirmativa Kopula
negative Kopula
Die Kopula »ש« verbindet Objektausdrücke und Eigenschafts- oder Klassenausdrücke zu ei- nem Satz, ebenso geordnete Paare von Gegenstandsausdrücken und zweistellige Beziehungs- ausdrücke, geordnete Tripel von Gegenstandsausdrücken und dreistellige Beziehungsaus- drücke - z. B.: »Jürgen ist Element der Klasse der Begabten « - »j $\varepsilon \lambda \times(\mathrm{x} \varepsilon \mathrm{B}) \ll ;$
"Stephan ist verliebt in Carola « - »<s, c $>\varepsilon$ Lb« (wobei »s « für »Stephan«, »c« für »Carola« und "Lb« für »verliebt in« steht).

\section{Junktoren (Aussagenlogik)}

$\begin{array}{lll}\neg & \text { nicht (Negation) } & \text { Negator } \\ \hat{\vee} & \begin{array}{l}\text { und (Konjunktion) } \\ \text { oder (nicht ausschließendes »oder«) } \\ \text { (Adjunktion oder Disjunktion) }\end{array} & \text { Konjunktor } \\ \supset, \rightarrow & \text { wenn - dann (Implikation oder Subjunktion) } & \text { Adjunktor oder Disjunktor } \\ \equiv, \leftrightarrow & \text { genau dann, wenn (Äquivalenz oder Bisubjunktion) } & \begin{array}{l}\text { Implikator/Subjunktor } \\ \text { Äquivalentor/Bisubjunktor/ } \\ \text { Biimplikator }\end{array}\end{array}$

weitere logische Konstante

$\neg, \leftrightarrow \quad$ entweder - oder, i. S. v. ausschließendes »oder«

(Kontravalenz)

Kontravalentor

\section{Quantoren (Quantoren- oder Prädikatenlogik)}
$\wedge, \forall$
alle
$\vee, \exists$
z. B.: $\forall_{x}-z u$ lesen als: für alle $x$ gilt
es gibt mindestens/einige
Allquantor
z. B.: $\exists_{\mathrm{x}}-\mathrm{zu}$ lesen als: es gibt mindestens ein $\mathrm{x}$,
Existenzquantor
für das gilt ...

\section{Folgerungssymbole}

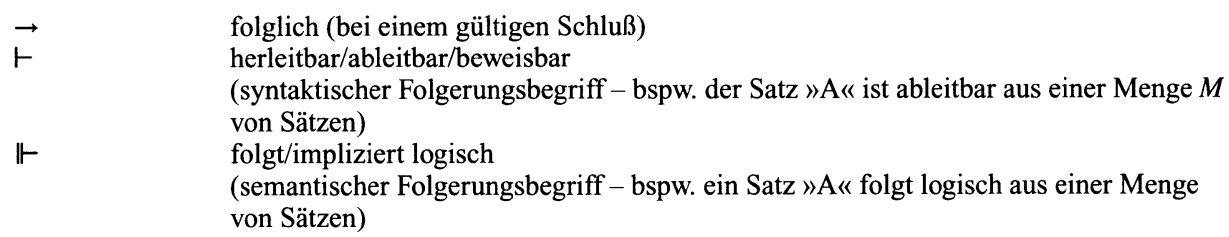




\section{Regel- und Kalkülsymbole}

$\begin{array}{ll}\Rightarrow & \text { es ist erlaubt, von ... überzugehen zu ... } \\ \Leftrightarrow & \text { es ist erlaubt, von ... überzugehen zu ... und umgekehrt } \\ =\mathrm{df}, \leftrightharpoons,:= & \text { nach Definition gleich }\end{array}$

\section{Relationssymbole}

$\begin{array}{ll}= & \text { gleich } \\ \neq & \text { nicht gleich } \\ \equiv & \text { identisch } \\ \sim & \text { äquivalent } \\ < & \text { kleiner } \\ \leq & \text { kleiner oder gleich } \\ > & \text { größer } \\ \geq & \text { größer oder gleich }\end{array}$

\section{Modallogik}

$\diamond$

es ist möglich, $\mathrm{da} \beta$

z.B.:

S - Sokrates war ein Philosoph

$\diamond \mathrm{S} \quad-$ es ist möglich, daß Sokrates ein Philosoph war

$\diamond \neg S \quad$ - es ist möglich, daß S. kein Philosoph war

$\neg \diamond \mathrm{S} \quad-$ es ist nicht möglich, daß S. ein Philosoph war

$\neg \diamond \neg S-$ es ist nicht möglich, daß S. kein Philosoph war

es ist notwendig, daß

$\Rightarrow, \rightarrow$

strikte Implikation: $\mathrm{p} \Rightarrow \mathrm{q}=\mathrm{df} \square(\mathrm{p} \rightarrow \mathrm{q})$

z. B.: wenn (im Straßenverkehr) ein Auto die Vorfahrt hat, dann hat es notwendigerweise das von rechts kommende Auto

$\Vdash$

semantische (modelltheoretische) Erfüllungsrelation

\section{Syllogistik}

\section{$\mathrm{S}$}

\section{Mengenlehre}

$<x, y>$
$\{x, y\}$
$\lambda$

\author{
Subjekt \\ Prädikat \\ universell bejahend (affirmo universaliter) \\ universell verneinend (nego universaliter) \\ partiell bejahend (affirmo partialiter) \\ partiell verneinend (nego partialiter)
}

geordnetes Paar

Paarmenge mit Elementen $\mathrm{x}, \mathrm{y}$

die Klasse der / Inbegriff der

z. B.: » $\lambda \times(x \in P h) \ll-z u$ lesen als: »die Klasse der Philosophierenden«

derjenige, diejenige, dasjenige; gibt es einen und nur einen Gegenstand, der die Eigenschaft $F$

hat, dann wird er durch den Ausdruck »t x(x $\varepsilon$ F)« gekennzeichnet - zu lesen als: »dasjenige

Ding, das F ist $\ll$

Element von

nicht ein Element von 
$\subset$

$\subseteq$

$\emptyset,\{\}$

$\cup$

$\hat{M}$

$M \backslash N$

$\mathrm{f}: \mathrm{M} \rightarrow \mathrm{N}$

$\omega$

$\mathbb{N}$

$£$

echte Menge von $-\mathrm{z}$. B.: $\mathrm{M} \subset \mathrm{N}-\mathrm{M}$ ist echte Menge von $\mathrm{N}$

Teilmenge von / eingeschlossen in / enthalten in

z. B.: »F $\subseteq \mathrm{G} «-$ zu lesen als: »Die Klasse/Menge $F$ ist Teilklasse/Teilmenge von $G »$ leere Menge

Vereinigung von $\ldots$ und ...

Durchschnitt von ... und ...

Differenzmenge/-klasse

$\mathrm{f}$ ist eine Abbildung von $\mathrm{M}$ in $\mathrm{N}$

kleinste unendliche Zahl

Menge der Natürlichen Zahlen

formale Sprache erster Stufe 